Research Article

\title{
Optimization Study of the Dump Diffuser in Gas Turbine to Reduce Pressure Loss
}

\author{
Fei Xing, Hao Su, Shining Chan (D), Leilei Xu, and Xinyi Yu \\ School of Aerospace Engineering, Xiamen University, No. 422, Siming South Road, Siming District, Xiamen, Fujian 361005, China \\ Correspondence should be addressed to Shining Chan; chansn2007@163.com
}

Received 23 December 2017; Revised 20 March 2018; Accepted 15 April 2018; Published 18 July 2018

Academic Editor: Linda L. Vahala

Copyright ( 2018 Fei Xing et al. This is an open access article distributed under the Creative Commons Attribution License, which permits unrestricted use, distribution, and reproduction in any medium, provided the original work is properly cited.

\begin{abstract}
As a key component-connecting compressor and the entrance of combustion chamber, the diffuser is able to increase the pressure and slow down the airflow in order to promote efficient combustion as well as avoid a large amount of pressure loss. In this paper, experimental investigation and numerical studies have been carried out to understand the effects of air bleeding from dump region and dump gap ratio on the total pressure loss and static pressure recovery of the dump diffusers. The ultimate objective is optimizing the dump diffuser design to get the maximum static pressure recovery and minimum total pressure loss. A simplified test model is used to study the effect of the air bleeding from the outer dump region and the dump gap ratio on the total pressure loss and static pressure recovery in the dump diffuser. The impact of the dump gap ratio in the performance of the dump diffusers has also been discussed. Nearly all the pressure raise occurs in the prediffuser, and most of the total pressure loss occurs in the dump region. For the recirculating area in the dump region, the controllable vortex can be introduced. Bleeding air from the outer dump region can improve the velocity distribution near the flame tube. The results show that when $0.4 \%$ of the air is bled from outer dump region, the performance of the dump diffuser is optimal. Hence, the controllable vortex method is effective for improving the performance of the dump diffuser.
\end{abstract}

\section{Introduction}

The performance of an aircraft cannot be improved without the support of an aeroengine which directly determines the flight characteristic, and the combustor is one of the three key components of aeroengines, which directly affects the engine performance. With high-performance compressor, the compressed air at compressor outlet is accelerated to $170 \mathrm{~m} / \mathrm{s}$ or higher. If the compressed air flows into the flame tube without being decelerated, it may cause serious problems on combustion stability and high total pressure loss. The gas turbine diffuser system is used to resolve the problems listed above. It is employed to decelerate the compressor discharge flow and distribute the air around the flame tube uniformly and stably. The diffusion process must be accomplished with minimum total pressure loss because these parasitic losses have an adverse effect on the thermal efficiency of the whole engine.

The dump diffuser is designed well to meet with the demand of the annual combustor in the current advanced aeroengines and commonly used. It can get a greater expansion ratio with a same length remained compared with the faired/aerodynamic diffuser. The compressor discharge flow is decelerated in a short, conventional prediffuser and then "dumped" into a large chamber, which divides the flow to the flame tube. Usually, the prediffuser is a typical pneumatic tunnel, and the airflow from the prediffuser outlet is divided into three parts. One part goes into the flame tube, and the other parts, respectively, flow into the combustor's inner and outer annulus flow path. The sudden expansion at the prediffuser existence causes the flow recirculation in the dump zone, which helps to maintain a stable flow pattern rather sensitive to the engine operation conditions. But the sudden expansion causes pressure losses, and the recirculation area makes the loss larger than in the aerodynamic diffuser.

Experimental and numerical studies have been focusing on the design of optimization in modern aerogas turbine engines during the last few decades. Those studies demonstrated that the performance of dump diffusers depends 


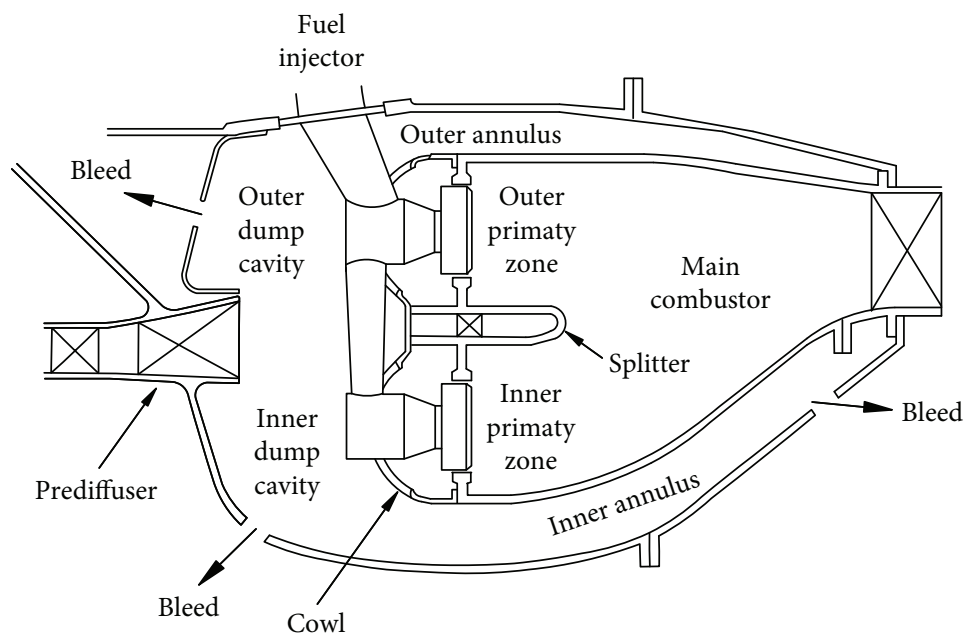

Figure 1: Bleeding program.
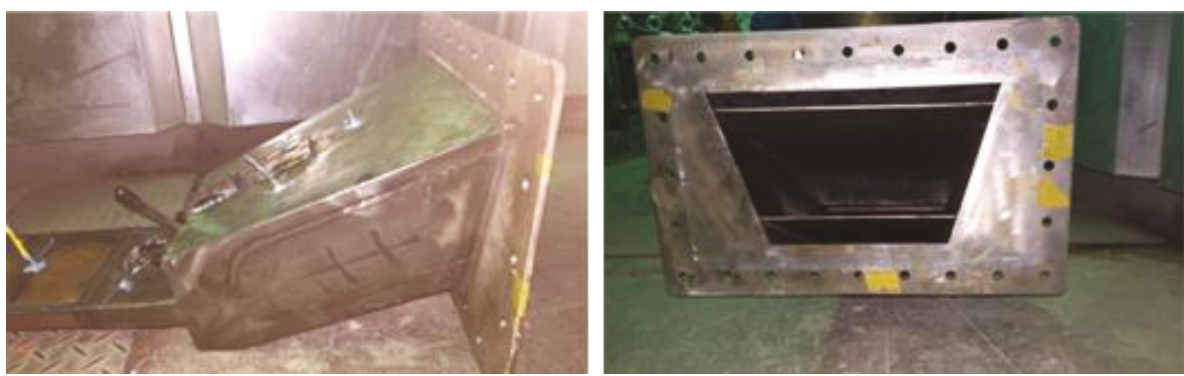

FIgURE 2: Test region of dump diffuser.

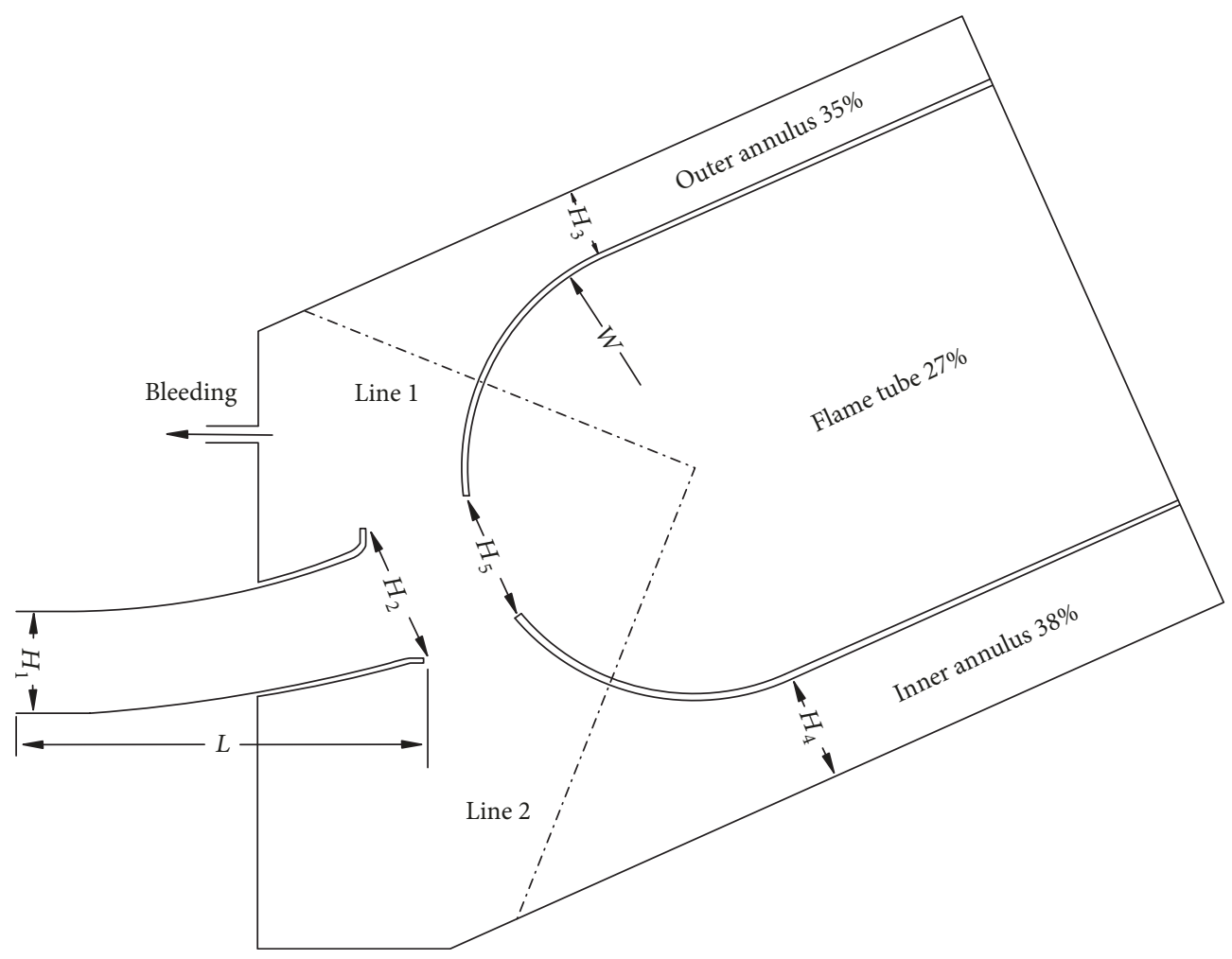

Figure 3: Idealized physical model of dump diffuser. 
TABle 1: Operating conditions $\left(H_{2} / H_{1}=1.73, T=30^{\circ} \mathrm{C}\right)$.

\begin{tabular}{lcccccccc}
\hline & Case 1 & Case 2 & Case 3 & Case 4 & Case 5 & Case 6 & Case 7 & Case 8 \\
\hline Inlet flow rate $(\mathrm{kg} / \mathrm{s})$ & 0.918 & 0.918 & 0.918 & 0.918 & 0.918 & 0.918 & 0.918 & 0.918 \\
Inlet pressure $(\mathrm{kPa})$ & 241.0 & 241.6 & 241.6 & 241.0 & 241.0 & 240.7 & 240.9 & 239.0 \\
Bleeding pressure $(\mathrm{kPa})$ & 243.7 & 239.6 & 235.2 & 228.2 & 220.8 & 210.4 & 198.6 & 186.2 \\
Bleeding percentage $(\%)$ & 0.00 & 0.33 & 0.49 & 0.64 & 0.77 & 0.88 & 0.97 & 1.04 \\
\hline
\end{tabular}

TABLe 2: Operating conditions $\left(H_{2} / H_{1}=1.73, T=150^{\circ} \mathrm{C}\right)$.

\begin{tabular}{lcccccccc}
\hline & Case 1 & Case 2 & Case 3 & Case 4 & Case 5 & Case 6 & Case 7 & Case 8 \\
\hline Inlet flow rate $(\mathrm{kg} / \mathrm{s})$ & 1.19 & 1.19 & 1.19 & 1.19 & 1.19 & 1.19 & 1.19 & 1.19 \\
Inlet pressure $(\mathrm{kPa})$ & 375.00 & 373.22 & 373.52 & 375.59 & 372.23 & 372.33 & 371.54 & 370.25 \\
Bleeding pressure $(\mathrm{kPa})$ & 380.94 & 375.34 & 375.26 & 362.89 & 356.68 & 342.58 & 330.89 & 307.59 \\
Bleeding percentage $(\%)$ & 0.00 & 0.29 & 0.44 & 0.56 & 0.69 & 0.80 & 0.90 & 0.96 \\
\hline
\end{tabular}

TABLE 3: Operating conditions $\left(H_{2} / H_{1}=1.20, T=30^{\circ} \mathrm{C}\right)$.

\begin{tabular}{lcccccccc}
\hline & Case 1 & Case 2 & Case 3 & Case 4 & Case 5 & Case 6 & Case 7 & Case 8 \\
\hline Inlet flow rate $(\mathrm{kg} / \mathrm{s})$ & 0.877 & 0.877 & 0.877 & 0.877 & 0.877 & 0.877 & 0.877 & 0.877 \\
Inlet pressure $(\mathrm{kPa})$ & 249 & 249 & 249 & 249 & 249 & 249 & 249 & 249 \\
Bleeding pressure $(\mathrm{kPa})$ & 250.9 & 247.6 & 241.9 & 234.5 & 227.4 & 216.4 & 205.7 & 194.2 \\
Bleeding percentage $(\%)$ & 0.00 & 0.36 & 0.53 & 0.68 & 0.83 & 0.95 & 1.05 & 1.13 \\
\hline
\end{tabular}

TABle 4: Operating conditions $\left(H_{2} / H_{1}=1.20, T=150^{\circ} \mathrm{C}\right)$.

\begin{tabular}{lcccccccc}
\hline & Case 1 & Case 2 & Case 3 & Case 4 & Case 5 & Case 6 & Case 7 & Case 8 \\
\hline Inlet flow rate $(\mathrm{kg} / \mathrm{s})$ & 1.096 & 1.096 & 1.096 & 1.096 & 1.096 & 1.096 & 1.096 & 1.096 \\
Inlet pressure $(\mathrm{kPa})$ & 364 & 364 & 364 & 364 & 364 & 364 & 364 & 364 \\
Bleeding pressure $(\mathrm{kPa})$ & 365.9 & 362.6 & 357.5 & 350.2 & 344.7 & 334.5 & 320.6 & 308.7 \\
Bleeding percentage $(\%)$ & 0.00 & 0.30 & 0.45 & 0.58 & 0.72 & 0.82 & 0.93 & 1.01 \\
\hline
\end{tabular}

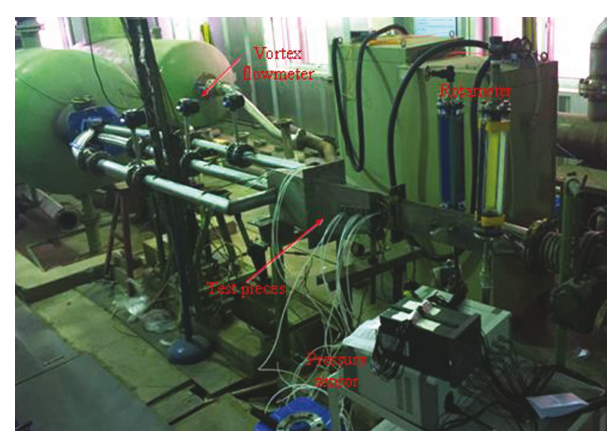

FIgURE 4: Experimental facility.

largely on the profile of prediffuser, the flow field of diffuser inlet, and the distance between the head of flame tube and the outlet of prediffuser. Fishenden and Stevens [1] studied the influence of the dump diffuser system. A full annular model of the dump diffuser is tested. The results show that the presence of flame tube has a beneficial effect on the performance and stability of flow in the prediffuser. They have concluded that the major static pressure rise occurs in the prediffuser portion, but most of the total pressure loss occurs in the dump region. The principal determinants of the total pressure loss are amount of diffusion being attempted downstream of the prediffuser and size and shape of the flame tube and the dump gap. Stevens et al. [2, 3], Klein $[4,5]$, Zierer [6], and Barker and Carrotte $[7,8]$ have considered how the interaction between the compressor and the operation conditions affects the downstream diffuser. That is, the annular diffuser can achieve a considerably higher area ratio and pressure recovery with the compressor-generated inlet conditions compared with those generated by a more classical ducted flow. Carrotte et al. [9] investigated the distance between the prediffuser and the flame tube, called dump gap. The results indicate that the dump gap could affect the flow distribution and the total pressure loss. The influence of the dump gas was also investigated by Hestermann et al. [10] and Honami et al. [11]. They optimized distance and showed the positive effect of the flame tube head on the upstream diffuser flow. Walker et al. [12] tested the interaction between the compressor outlet guide vane (OGV) and prediffuser. The results demonstrate that, as the dump gap increases, the component interactions decrease. 


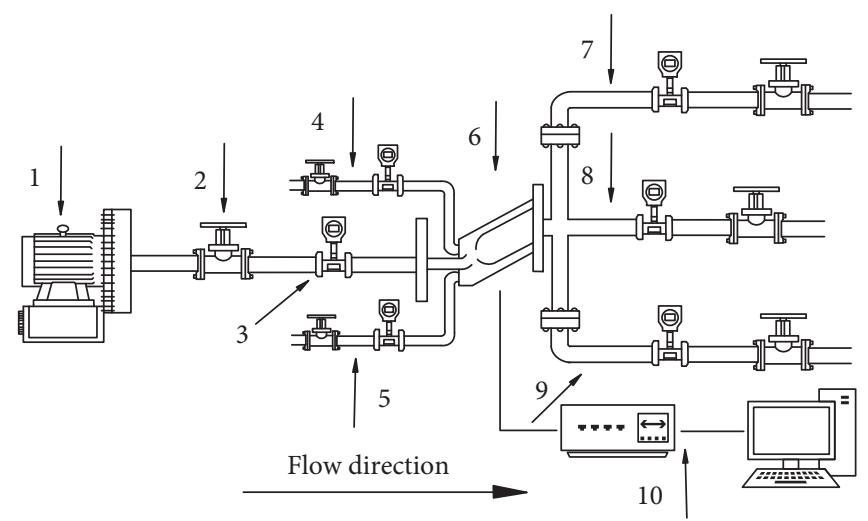

Figure 5: Test system schemes: (1) compressor, (2) main valve, (3) vortex street flowmeter, (4) rotameter of outer annulus flow path, (5) rotameter of inner annulus flow path, (6) dump diffuser, (7) outer annulus flow path flow control, (8) flame tube flow control, (9) the inner flow control, and (10) data acquisition system.

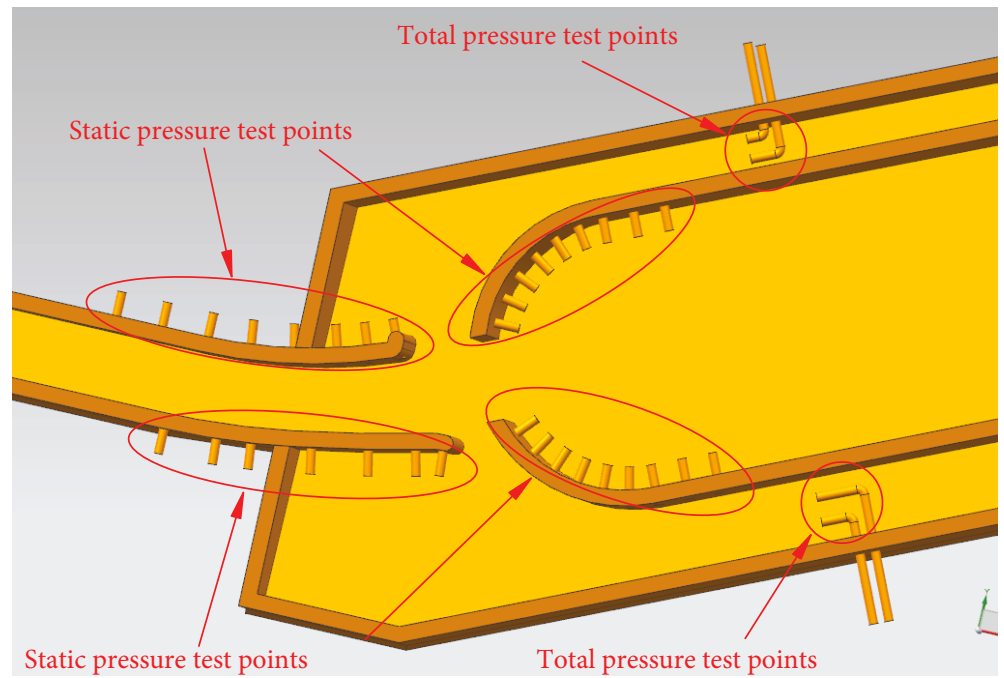

FIGURE 6: The total and static pressure test points.
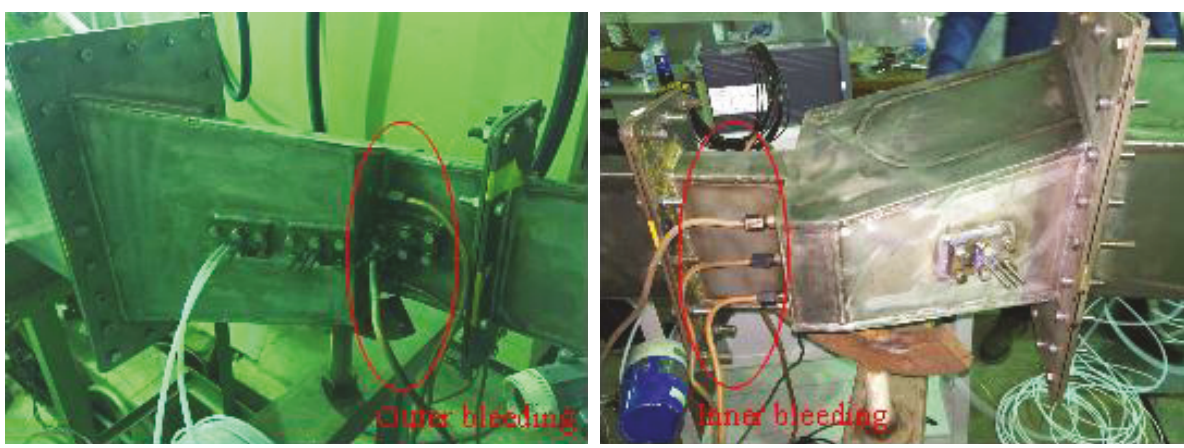

FIGURE 7: The outer and inner bleeding hole.

TABle 5: Experimental flow parameters.

\begin{tabular}{lcccccc}
\hline Working conditions & Pressure $(\mathrm{kPa})$ & Temperature $(\mathrm{K})$ & Density $\left(\mathrm{kg} / \mathrm{m}^{3}\right)$ & Mach & Reynolds & Flow rate $(\mathrm{kg} / \mathrm{s})$ \\
\hline Actual & 469 & 512 & 3.19 & 0.27 & 542193.3 & 1.2 \\
Experimental & 231 & 287 & 2.80 & 0.27 & 542659.9 & 0.865 \\
\hline
\end{tabular}




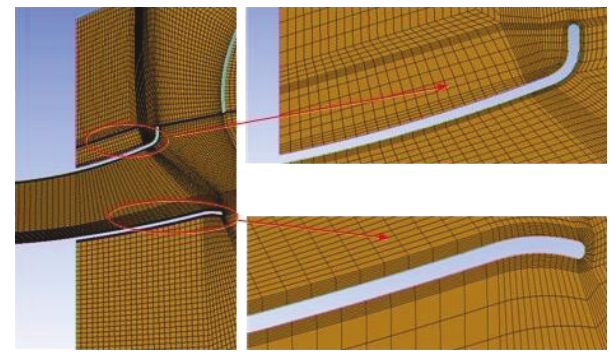

FIGURE 8: Sample of multiblock-structured mesh at dump region.

At a dump gap ratio of 0.8 , the proximity of the flame tube influences the prediffuser providing a beneficial blockage effect. However, if increased to 1.2, this beneficial effect is weakened and the prediffuser flow deteriorates. Sanalkumar et al. [13] demonstrated the relation between the combustor walls and the total pressure loss. The author presented an idealized physical model of a dump diffuser after calculated the static pressure distribution on the upper walls of diffusers with the same dump gap distance but different combustor walls. Karki et al. [14] analyzed the dump diffuser by using computational procedure. The procedure is based on the solution of the Navier-Stokes equations on a body-conforming grid. The turbulence effects are simulated using the high Reynolds number form of the $k-\varepsilon$ model. The estimates provided by the simplified model for static pressure recovery and total pressure losses are within $10 \%$ and $15 \%$ of the experimental data.

In order to improve the performance of dump diffuser, the controllable vortex diffuser has been studied. Juhasz [15] studied a short dump diffuser with bleeding method, and the experimental results showed when $3.7 \%$ of air bled out from the inner wall, the airflow is completely attached to the inner wall; and when $6.1 \%$ of air bled out from the outer wall, the airflow is completely attached to the outer wall. It can get different outlet velocity distribution while using different proportions to bleed air from inner and outer wall. It means that the bleeding could control the outlet velocity distribution and then control the flow distribution. The experiments also proved that bleeding could also reduce the total pressure loss. Lefebvre [16] studied the combustion performance of the combustor assembling the dump diffuser with bleeding in the dump region (Figure 1). The experimental results show that, compared to conventional diffuser, the dump diffuser could reduce $40 \%$ of NOx emissions. Xu et al. [17] studied the influence of prediffuser wall angle and prediffuser length on the performance of dump diffusers and identified the pressure loss mechanisms. It was observed that apparent flow separation occurred on prediffuser wall when prediffuser wall angle amplified to certain degree. The prediffuser exit flow was distorted, indicating that the uniform exit conditions typically assumed in the diffuser design were violated. Skew distribution of the prediffuser outlet flow can result in strong transverse mixing for liquid; the total pressure loss of prediffuser increases significantly. The formula of the total pressure loss coefficient and the pressure recovery coefficient was developed.
The optimal prediffuser length and prediffuser wall angle can be conveniently obtained by this equation; the calculation error was less than $5 \%$.

He et al. [18] have investigated the influence of the dump gap on aerodynamic performance of a diffuser. The dump gap ratio varies from 0.8 to 3.5 . And the diffusion loss into the cowl was measured. Thus, the real overall performance of the diffuser system can be obtained. They had come to the conclusion that the loss coefficient of the prediffuser decreases as the dump gap increases. The overall loss coefficient is relatively high when the dump gap ratio is smaller than 1.2 or larger than 2.8 and is relatively low and insensitive to dump gap with intermediate dump gaps. Shen et al. [19] presented numerical simulation of a combustor diffusion system of a full-scale single annular combustor, mainly focusing on the effects of the cowling geometry, the area ratio of the prediffuser, and the axial length of the dump gap. Sathyan et al. [20] have carried out parametric analytical studies for examining the aerodynamic characteristics of different dump diffusers for modern aircraft engines. Numerical studies have been conducted using SST $k-\omega$ turbulence model. It is concluded that in addition to the dump gap ratio, the aerodynamic shape of the flame tube case and the other geometric variables are also needed to be optimized judiciously after considering the fluid dynamic constraints for controlling the pressure recovery and the losses. Ghose et al. [21] have studied the effects of different prediffuser angles, inlet swirl levels, and different dump gaps on the streamline distribution and the static pressure recovery coefficients. They observed that the dump gap has significant influence on the static pressure recovery only at small prediffuser angle. Bhat et al. [22] described the effect of diffuser geometry on the performance parameters of short annular combustors. They observed that swirl level in the inlet flow considerably influences the pressure recovery. As the inlet swirl level increases, the size of the corner recirculation decreases, but a central recirculation is additionally formed. The static pressure recovery increases with the inlet swirl level in the range of swirl number considered.

Prior to this, the work has been undertaken to design diffusers with greater prediffuser wall angle and the flow separation. The expansion of prediffuser wall angle can decrease the length of the prediffuser efficiently, as well as decrease the total pressure loss. In this paper, PIV experiments are conducted with a simplified test model to help choosing the congruent turbulence model, and the main objective of the cold flow test is to learn the influence of the bleeding air from outer annulus flow path in dump region.

\section{Experiment Description}

2.1. Physical Model. The experimental diffuser model is manufactured by stainless steel, which comprises a prediffuser followed by a dump diffuser and a flame tube (Figure 2). To guarantee the smoothness of inlet airflow, a straight section is installed before the entrance of the prediffuser. An idealized physical model of dump diffuser is shown in Figure 3. Experiments are conducted at $30^{\circ} \mathrm{C}$ and $150^{\circ} \mathrm{C}$ conditions, 



FIGURE 9: Experimental facility.

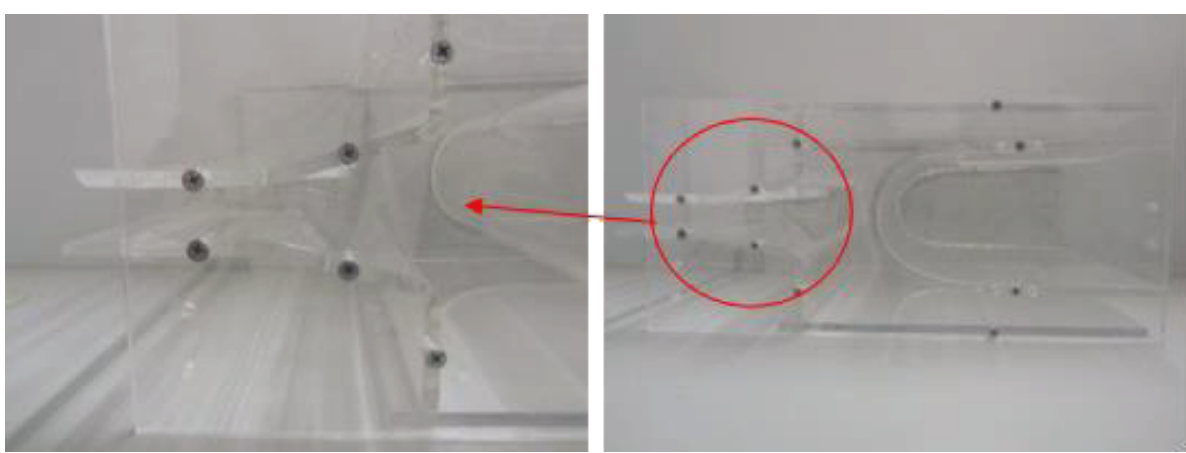

Figure 10: Test region of dump diffuser.

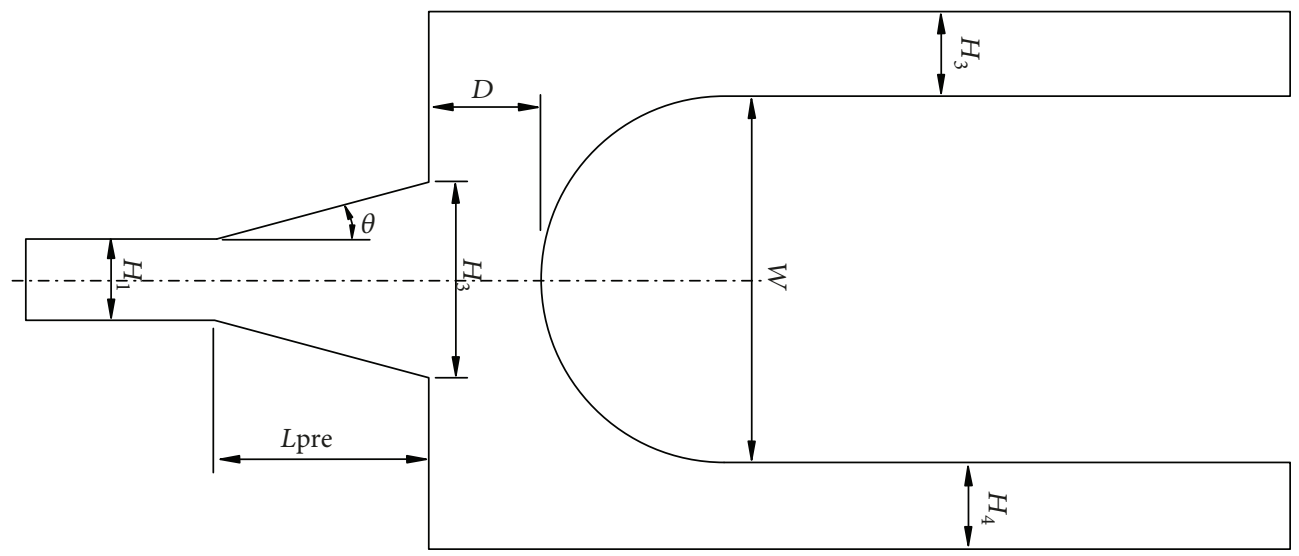

FIGURE 11: Idealized physical model of dump diffuser with straight-walled prediffuser.

leading the prediffuser expansion ratio $H_{2} / H_{1}=1.20$ and 1.73. Bleeding program is shown in Tables $1-4$.

2.2. Experimental Setup. The present research is carried out in the Engine Combustion Laboratory of Nanjing University of Aeronautics and Astronautics (Figure 4). The whole setup includes a single screw air compressor, flow-regulating valves, vortex street flowmeters, thermocouples, pressureregulating valve, and pressure control valve. Figure 5 shows the test system schematic diagram. The total inlet flow and the flow in annulus flow path of dump diffuser are separately controlled by the main valve and rotameters. The distribution of diffuser's outlet flow is controlled by three vortex street flowmeters: the outer annulus flow path is $35 \%$, the flame tube channel is $27 \%$, and the inner annulus flow path is $38 \%$. The total pressure test points and static pressure test points are shown in Figure 6.

There are three $4 \mathrm{~mm}$ holes on the wall of the outer dump region (Figure 7). Float flowmeters with high precision are used to guarantee that the bleeding from holes is $1 \%$ out of total intake air.

2.3. Experimental Procedure. Due to the compressor, the inlet flow of diffuser is of the high temperature and pressure. For example, the inlet pressure, temperature, and Mach number of the diffuser are $469 \mathrm{kPa}, 512 \mathrm{~K}$, and 0.27 , respectively. Such 


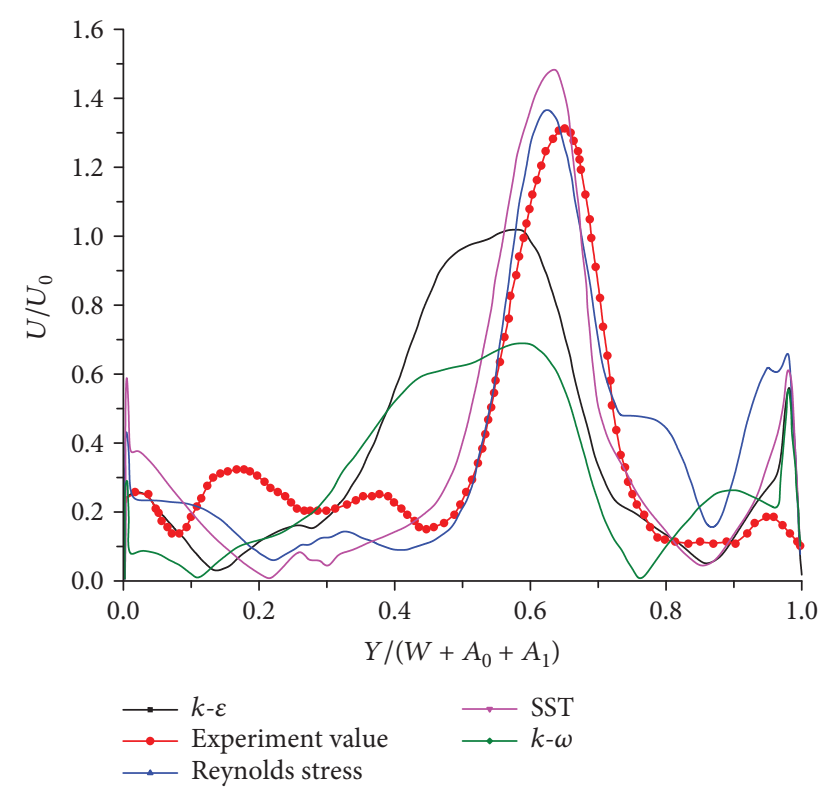

Figure 12: Comparison of predicted and measured velocity in dump diffuser.

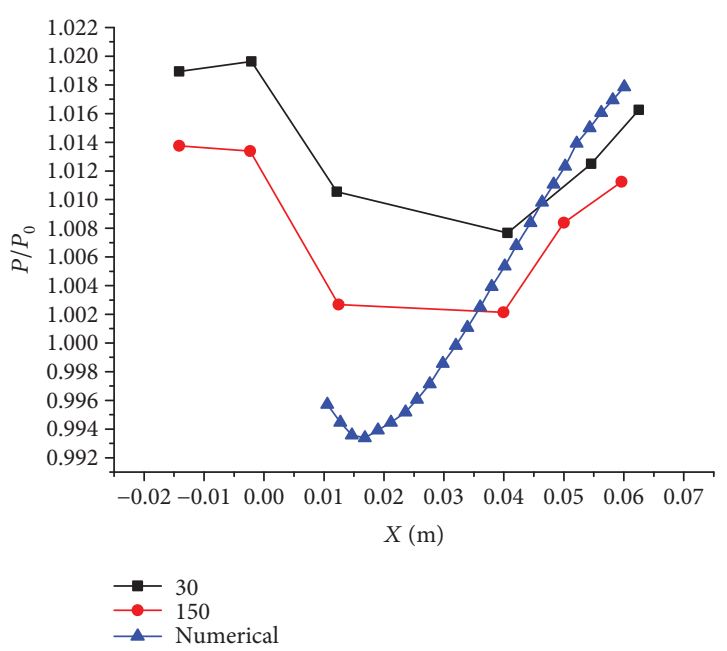

FIGURE 13: Pressure distribution on the top wall of prediffuser.

operating conditions are very difficult to achieve in the laboratory. Taking safety and economic efficiency into consideration, the hydrodynamic similarity criterion law is used to ensure consistent inlet Mach number and Reynolds number. The inlet parameters which are shown in Table 5 are determined by the experimental conditions.

Prediffuser expansion ratio is set to 1.20 and 1.73. The inlet flow is controlled by the main valve; the outlet flow is controlled by vortex flow meter and a valve: the flow that goes through the outer annulus flow path is $35 \%$ out of the inlet flow, the flame tube channel is $27 \%$, and the inner annulus flow path is $38 \%$. Tests with and without bleeding are performed under both temperatures of $30^{\circ} \mathrm{C}$ and $150^{\circ} \mathrm{C}$. During the bleeding test, the effect of increasing amount of outflow on the total pressure loss and the static pressure recovery is observed.

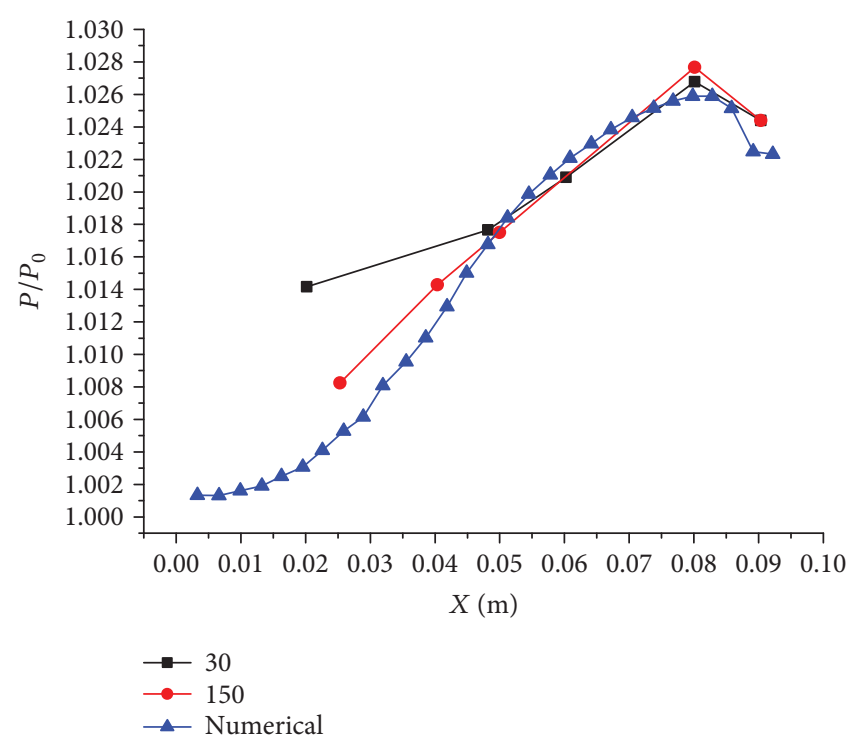

Figure 14: Pressure distribution on the under wall of prediffuser.

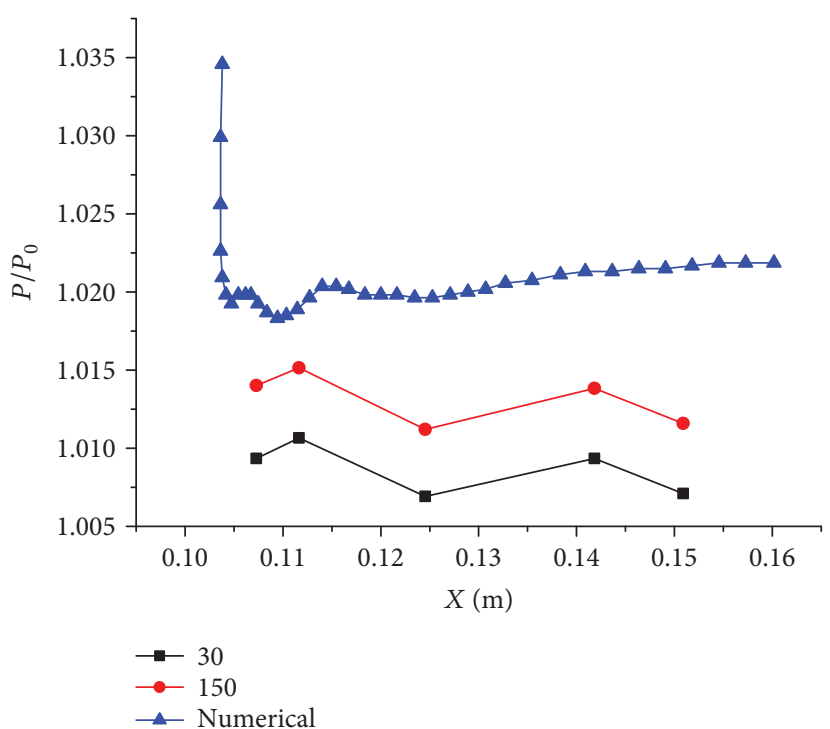

Figure 15: Pressure distribution on the top wall of flame tube.

Probes with diameter of $1.5 \mathrm{~mm}$ are used to measure the static pressure and the total pressure of the inlet and outlet of the diffuser in order to calculate the total pressure loss and the static pressure recovery.

For the bleeding test, the dimensionless pressure of the test data will be used as the ordinate axis, namely, the entrance static pressure as the reference pressure. The flow direction of the horizontal axis is the same.

2.4. Data Reduction. Only the wall pressure of diffuser is measured during the experiment. When calculating the static pressure recovery coefficient, pressure on the straight section of the outer annulus channel is the outer outlet section pressure, and pressure on the straight section of the inner annulus channel is the inner outlet section pressure. 


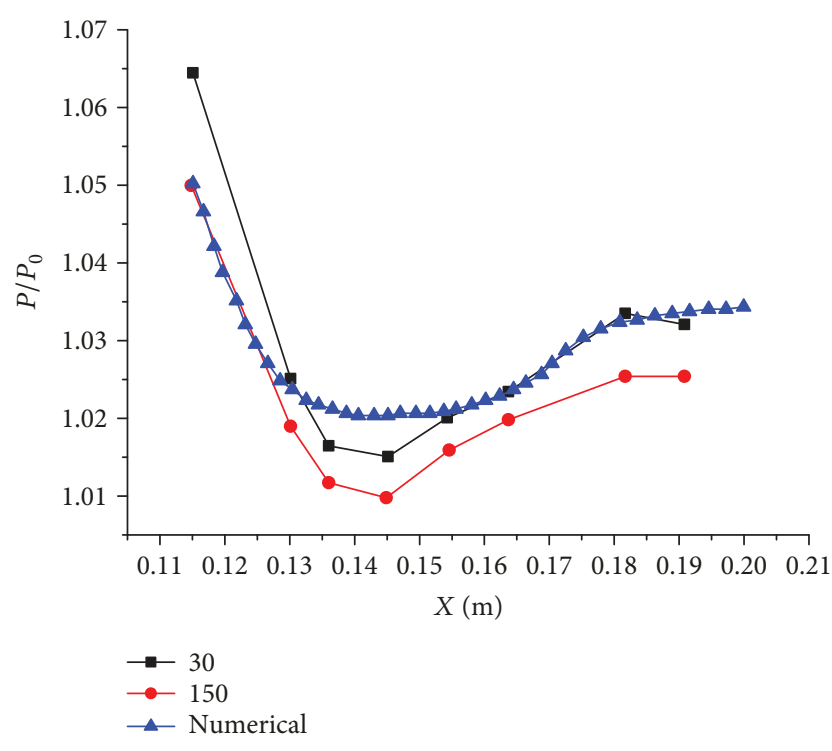

FIgURe 16: Pressure distribution on the under wall of flame tube.

The total pressure of outlet of inner and outer annulus flow path is calculated as

$$
P_{\mathrm{t}}=P+0.5 \times \rho \times V^{2},
$$

while the velocity is

$$
V=\frac{m}{A \times \rho},
$$

where $m$ is the mass flow rate, the inner flow accounts for $38 \%$ of the total flow, and the outer flow accounts for $35 \%$.

The static pressure recovery coefficient is defined as

$$
C_{\mathrm{p}}=\frac{0.35 \times P_{\text {out }}+0.38 \times P_{\text {in }}+0.27 \times P_{\text {flame }}-P_{0}}{0.5 \times \rho \times V_{0}^{2}} .
$$

The total pressure loss coefficient is

$$
\Omega=\frac{P_{\mathrm{t} 0}-0.35 \times P_{\text {tout }}-0.38 \times P_{\text {tin }}-0.27 \times P_{\text {flame }}}{P_{\mathrm{t} 0}} .
$$

\section{Numerical Model and Scheme}

3.1. Numerical Model. The performance of dump diffuser with air bleeding is studied. Geometrical parameters of diffuser are as follows: $H_{1}=21.6 \mathrm{~mm}, H_{2}=30 \mathrm{~mm}, H_{3}=14.6$ $\mathrm{mm}, H_{4}=22.8 \mathrm{~mm}, H_{5}=27.4 \mathrm{~mm}, W=49 \mathrm{~mm}$, and $L=$ $86.9 \mathrm{~mm}$; the expansion gap ratio is 1 , the distance to axis is $219.8 \mathrm{~mm}$, and the prediffuser expansion ratio is 1.5. As the flow distribution, the inner flow accounts for $38 \%$ of the total flow, the outer flow accounts for $35 \%$, and the flame tube accounts for $27 \%$.

3.2. Numerical Setup. In this paper, the effects of bleeding on the performance of dump diffuser are explored by threedimensional steady-state CFD simulations. Please see Figure 3 for the geometry scheme of the diffuser for calculation, and Figure 8 shows the sample of multiblock-structured mesh with close views at dump region. The mesh quality is commonly assessed with determinant and angle. Determinant calculated Jacobian determinant for each six-sided and then standardized the matrix of determinant to characterize the deformation of the unit. The value 1 indicates an ideal hexahedron cube, while 0 indicates the transcube with negative volume, which is represented on the $x$-axis from 0 to 1 . Typically, the values over 0.3 are usually acceptable for most solvers. The smallest interior angle of each grid cell is checked for assessment, generally between $0^{\circ}$ and $90^{\circ} .90^{\circ}$ stands for the highest mesh quality; the general minimum angle should be greater than 18 degrees.

The key to meshing the prediffuser is the wall thickness, and there is a certain curve in the prediffuser outlet which needs to remove the wall block. The solution is to use the C-type grid and wall maps. To improve the mesh quality, O-type mesh is used to build the grid, which effectively improved the mesh quality and controlled the boundary wall of prediffuser.

3.3. Turbulence Model Sensitivity. In this paper, the turbulence model is determined by comparing the experimental results of the velocity distribution in the dump region with the results of the simulation using different turbulence models. The experiment is carried out in the towing tank of the "Fluid Mechanics and PIV Laboratory (FMPL)" in Xiamen University (Figure 9). The size of the test section is $0.5 \mathrm{~m} \times 0.58 \mathrm{~m} \times 2 \mathrm{~m}$. It consists of a towing water tank and a trolley supporting the towing system and the control unit. The towing speed can reach the maximum velocity of $0.5 \mathrm{~m} / \mathrm{s}$.

This PIV system is provided by Dantec. The PIV measurement system consists of five subsystems, that is, quasicontinuous laser light source, optical lens with mirror, one CMOS camera, tracer particles, and PIV software. The laser is installed on sideways of the flume, vertically under the test section, giving a light sheet parallel to the camera. The high speed CMOS camera is vertically mounted behind the tank to acquire the information of the plain which is illuminated by the light sheet. Then, the collected data is sent to computer for calculation.

PIV system is used to obtain the velocity field of a rectangle combustor in the nonreacting condition. The experimental model is shown in Figure 10, and it is manufactured by transparent acrylic boards. An idealized physical model of a dump diffuser with the straight wall prediffuser is shown in Figure 11. Baseline values $\left(H_{1}=\right.$ $21.7 \mathrm{~mm}, L_{\text {pre }}=57 \mathrm{~mm}, W=98 \mathrm{~mm}, H_{3}=H_{4}=20.5 \mathrm{~mm}$, $\theta=9^{\circ}$, and $D / H_{2}=1.02$ ) are based on a typical diffuser. Flow velocity is set to $0.25 \mathrm{~m} / \mathrm{s}$ [23].

In order to facilitate the comparative analysis, relative coordinate and velocity are used. The distance between the selected section and the diffuser outlet is $60 \%$ of the dump gap length. The prediction results using RSM are in good qualitative agreement with the experimental results, especially for the maximum velocity position and inflection points (Figure 12). The SST model also gives good predictions. However, the maximum velocity predicted by the SST model is slightly larger than the experimental data. The 
TABLE 6: Diffuser performance parameters $\left(H_{2} / H_{1}=1.73, T=30^{\circ} \mathrm{C}\right)$.

\begin{tabular}{lcccccccc}
\hline & Case 1 & Case 2 & Case 3 & Case 4 & Case 5 & Case 6 & Case 7 & Case 8 \\
\hline Inlet flow rate $(\mathrm{kg} / \mathrm{s})$ & 0.918 & 0.918 & 0.918 & 0.918 & 0.918 & 0.918 & 0.918 & 0.918 \\
Inlet pressure $(\mathrm{kPa})$ & 241.0 & 241.6 & 241.6 & 241.0 & 241.0 & 240.7 & 240.9 & 239.0 \\
Bleeding pressure $(\mathrm{kPa})$ & 243.7 & 239.6 & 235.2 & 228.2 & 220.8 & 210.4 & 198.6 & 186.2 \\
Bleeding percentage $(\%)$ & 0.00 & 0.33 & 0.49 & 0.64 & 0.77 & 0.88 & 0.97 & 1.04 \\
$\Omega(\%)$ & 2.58 & 2.63 & 2.49 & 2.62 & 2.43 & 2.49 & 2.49 & 2.74 \\
$C_{\mathrm{p}}(\%)$ & 49.18 & 48.21 & 50.68 & 48.56 & 51.99 & 50.87 & 50.85 & 47.13 \\
\hline
\end{tabular}

TABLE 7: Diffuser performance parameters $\left(H_{2} / H_{1}=1.73, T=150^{\circ} \mathrm{C}\right)$.

\begin{tabular}{lcccccccc}
\hline & Case 1 & Case 2 & Case 3 & Case 4 & Case 5 & Case 6 & Case 7 & Case 8 \\
\hline Inlet flow rate $(\mathrm{kg} / \mathrm{s})$ & 1.19 & 1.19 & 1.19 & 1.19 & 1.19 & 1.19 & 1.19 & 1.19 \\
Inlet pressure $(\mathrm{kPa})$ & 375.00 & 373.22 & 373.52 & 375.59 & 372.23 & 372.33 & 371.54 & 370.25 \\
Bleeding pressure $(\mathrm{kPa})$ & 380.94 & 375.34 & 375.26 & 362.89 & 356.68 & 342.58 & 330.89 & 307.59 \\
Bleeding percentage $(\%)$ & 0.00 & 0.29 & 0.44 & 0.56 & 0.69 & 0.80 & 0.90 & 0.96 \\
$\Omega(\%)$ & 2.57 & 2.44 & 2.04 & 3.00 & 2.47 & 2.59 & 2.66 & 3.74 \\
$C_{\mathrm{p}}(\%)$ & 47.43 & 50.30 & 57.64 & 39.43 & 49.98 & 47.79 & 46.74 & 27.37 \\
\hline
\end{tabular}

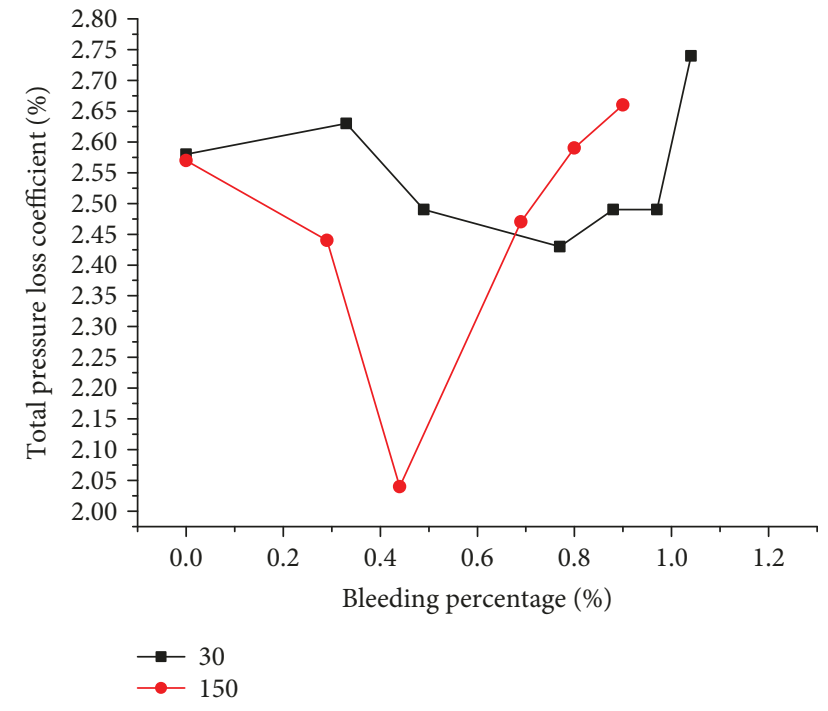

Figure 17: Relationship between the total pressure loss coefficient and the bleeding percentage (1.73 expansion ratio).

$k-\varepsilon$ and $k-\omega$ models have relatively large prediction errors in the flow field of diffuser. This is due to the fact that the $k-\varepsilon$ and $k-\omega$ models are based upon the turbulence viscosity hypothesis [24]. Simple turbulence viscosity assumption in the Boussinesq hypothesis cannot accurately capture the Reynolds stresses as well as the complex flow separation in the diffuser. Date [25] also reported that the turbulence viscosity models are insufficient for unrestricted flows with strong separation or far wakes. Problems are also encountered for flows with large extra strain such as curved boundary layers, diverging passages, and swirling flows where highly anisotropic turbulence has a dominant effect. The literature $[26,27]$ compared the predictions of total pressure loss of the different models; the result shows that $k-\varepsilon$ and $k-\omega$ models usually over predict the total pressure loss. Walker et al. [28, 29] reported that Reynolds stress model gives better agreement with measured data in a dump diffuser.

\section{Results and Discussion}

4.1. Experimental Validation. Experiments without air bleeding are carried out at $30^{\circ} \mathrm{C}$ and $150^{\circ} \mathrm{C}$, respectively. The wall pressure of prediffuser will be measured to compare with numerical results. The pressure distributions of the top and under walls of the prediffuser and the flame tube are presented in Figures 13-15, and 16.

As shown from Figures 13 and 14, the experimental data agree well with the numerical results at the prediffuser outlet. Figure 15 shows that there are 1-2\% deviations on the top wall of flame tube, and the stagnation point pressure cannot be measured. The pressure measured at other measuring points is consistent with the trend. Figure 16 shows a better pressure distribution on the under wall of flame tube. The pressure is highest at stagnation point, then decreases rapidly along the wall, and then slowly rises near the outlet of the flame tube. Two kinds of pressure distribution condition trends of the diffuser wall are basically the same. It can be seen that it has ensured the inlet Reynolds number and Mach number and lowered the temperature and pressure leading to reliable results.

In this experiment, the inlet flow is controlled by the main valve and the inlet pressure relies on controlled flow. There are some deviations about the flow rate and the pressure between the real situations with numerical results (Tables 6 and 7). When the inlet temperature is $30^{\circ} \mathrm{C}$, the inlet flow rate is $0.918 \mathrm{~kg} / \mathrm{s}$ which is $5.5 \%$ higher than the calculated value. When the inlet temperature is $150^{\circ} \mathrm{C}$, the 


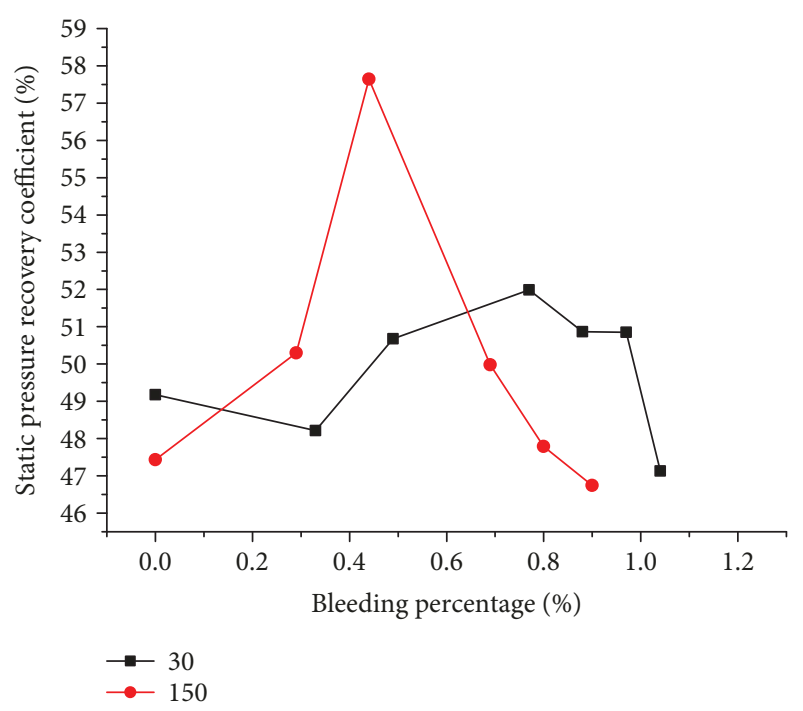

FIGURE 18: Relationship between the static pressure recovery coefficient and the bleeding percentage (1.73 expansion ratio).

inlet flow rate is $1.19 \mathrm{~kg} / \mathrm{s}$ which is $7 \%$ higher than the calculated value. There is a difference between experimental data and numerical results on inlet pressure because of fluctuations of airflow.

In order to observe more visually, the bleeding percentage is set to the $x$-axis, and the total pressure loss coefficient and the static pressure recovery coefficient are set to the $y$-axis. Figure 17 shows that with the increase of bleeding, the total pressure loss coefficient firstly decreases and then increases. The minimum appears at $0.4 \% \sim 0.7 \%$ of air bleeding. Figure 18 shows that the static pressure recovery coefficient firstly increases and then decreases. The maximum appears at $0.4 \% \sim 0.7 \%$ of air bleeding.

The performance parameters of the prediffuser with an expansion ratio of 1.20 are different, as shown in Tables 8 and 9. When the inlet temperature is $30^{\circ} \mathrm{C}$, the inlet flow rate is $0.877 \mathrm{~kg} / \mathrm{s}$, higher than the calculated value by $1 \%$. When the inlet temperature is $150^{\circ} \mathrm{C}$, the inlet flow rate is $1.096 \mathrm{~kg} / \mathrm{s}$, which is lower than the calculated value by $1.4 \%$.

As can be seen in Figures 19 and 20, the total pressure loss coefficient and the static pressure coefficient are optimal at the air bleeding percentage of $0.4 \%$ to $0.8 \%$. When air bleeding percentage is higher than $0.9 \%$, the total pressure loss increases rapidly, and the static pressure recovery coefficient decreases accordingly.

From the two cold flow test data above, we can see that increasing the expansion ratio of the prediffuser could effectively enhance the performance of the diffuser, which would reduce the total pressure loss and increase the static pressure recovery. When the bleeding percentage is constant, the total pressure loss coefficient of a diffuser with expansion ratio of 1.73 is $2.58 \%$ and the static pressure recovery coefficient is $49.18 \%$, while the total pressure loss coefficient of the diffuser with expansion ratio of 1.2 is $3.12 \%$ and the static pressure recovery coefficient is $30.68 \%$. The total pressure loss was decreased by $17.2 \%$, and the static pressure recovery coefficient was increased by $60.3 \%$.

The air bleeding improves the airflow situation at dump region in diffuser. For the diffuser with expansion ratio of $1.2,0.36 \%$ of bleeding air out of total airflow decreases the total pressure loss by $8 \%$ and raises the static pressure recovery coefficient by $16.6 \%$. As for the diffuser with expansion ratio of $1.73,0.44 \%$ of bleeding air out of total airflow decreases the total pressure loss by $20.8 \%$ and raises static pressure recovery coefficient by $21.54 \%$.

4.2. Numerical Results. Numerical calculation shows that the total pressure loss of the prediffuser without bleeding is $5.23 \%$, and the static pressure recovery coefficient is $30.44 \%$. The total pressure loss of the whole diffuser is $29.45 \%$ and the static pressure recovery coefficient is $35.09 \%$ and the total pressure loss in dump region is $82.24 \%$. As shown in Figure 21, there is a recirculation area after dump region. The presence of the recirculation area helps to resist inlet airflow from distortion. Larger recirculation area will compress the mainstream area, the wall velocity of uneven flame tube, and the total pressure loss increase. Thus, it is necessary to reduce the recirculation area and enlarge the mainstream area.

In order to study how the bleeding air effects the performance of diffuser, the bleeding air is set, respectively, at $0.1 \%$, $0.3 \%, 0.4 \%, 0.5 \%, 0.7 \%, 0.8 \%$, and $1 \%$ of the total intake air. Calculation results are shown in Figure 22 and Table 10. It can be seen that air bleeding from the dump region has little effect on the total pressure loss and the static pressure recovery of the prediffuser for it has no boundary layer separation. On contrast, the total pressure loss of dump region reduced from $24.22 \%$ to about $20 \%$, the static pressure recovery coefficient has doubled. Similar to the whole diffuser, its total pressure loss has decreased and the static pressure recovery coefficient increased. With the increase of air bleeding, the total pressure loss decreases first and then increases, and the optimum is at $0.4 \%$.

In order to explain the influence of bleeding air to the diffuser performance, a $45^{\circ}$ angle between inner and outer rings with axis of flame tube is chosen; flow direction is set as the $x$-axis and velocity distribution as the $y$-axis (see Figure 3).

Results show that the bleeding air from outer annulus path can effectively reduce the velocity of the mainstream area and make velocity distribution more uniform. When the air bleeding percentage is $4 \%$, the maximum velocity is minimal, which means that the velocity distribution of the outer annulus path is the most average. With the increase of air bleeding, the pressure of the outer dump region decreases and inner dump region moves up, which causes that the airflow through the outer annulus path has a higher proportion of the total airflow.

Results above show that the air bleeding from the dump region can improve the distribution of the flow field and make the velocity distribution near the flame tube more uniform and reduce the total pressure loss. When the bleeding percentage is $0.4 \%$, the diffuser has the best performance. 
TABLE 8: Diffuser performance parameters $\left(H_{2} / H_{1}=1.20, T=30^{\circ} \mathrm{C}\right)$.

\begin{tabular}{lcccccccc}
\hline & Case 1 & Case 2 & Case 3 & Case 4 & Case 5 & Case 6 & Case 7 & Case 8 \\
\hline Inlet flow rate $(\mathrm{kg} / \mathrm{s})$ & 0.877 & 0.877 & 0.877 & 0.877 & 0.877 & 0.877 & 0.877 & 0.877 \\
Inlet pressure $(\mathrm{kPa})$ & 249 & 249 & 249 & 249 & 249 & 249 & 249 & 249 \\
Bleeding pressure $(\mathrm{kPa})$ & 250.9 & 247.6 & 241.9 & 234.5 & 227.4 & 216.4 & 205.7 & 194.2 \\
Bleeding percentage $(\%)$ & 0.00 & 0.36 & 0.53 & 0.68 & 0.83 & 0.95 & 1.05 & 1.13 \\
$\Omega(\%)$ & 3.12 & 2.87 & 2.95 & 3.06 & 2.96 & 3.44 & 3.22 & 3.28 \\
$C_{\mathrm{p}}(\%)$ & 30.68 & 35.77 & 34.15 & 31.84 & 33.91 & 24.05 & 28.70 & 27.33 \\
\hline
\end{tabular}

TABLE 9: Diffuser performance parameters $\left(H_{2} / H_{1}=1.20, T=150^{\circ} \mathrm{C}\right)$.

\begin{tabular}{lcccccccc}
\hline & Case 1 & Case 2 & Case 3 & Case 4 & Case 5 & Case 6 & Case 7 & Case 8 \\
\hline Inlet flow rate $(\mathrm{kg} / \mathrm{s})$ & 1.096 & 1.096 & 1.096 & 1.096 & 1.096 & 1.096 & 1.096 & 1.096 \\
Inlet pressure $(\mathrm{kPa})$ & 364 & 364 & 364 & 364 & 364 & 364 & 364 & 364 \\
Bleeding pressure $(\mathrm{kPa})$ & 365.9 & 362.6 & 357.5 & 350.2 & 344.7 & 334.5 & 320.6 & 308.7 \\
Bleeding percentage $(\%)$ & 0.00 & 0.30 & 0.45 & 0.58 & 0.72 & 0.82 & 0.93 & 1.01 \\
$\Omega(\%)$ & 3.03 & 3.08 & 3.05 & 3.14 & 2.91 & 3.31 & 3.37 & 3.29 \\
$C_{\mathrm{p}}(\%)$ & 33.77 & 32.81 & 33.38 & 31.48 & 36.16 & 28.18 & 26.91 & 28.57 \\
\hline
\end{tabular}

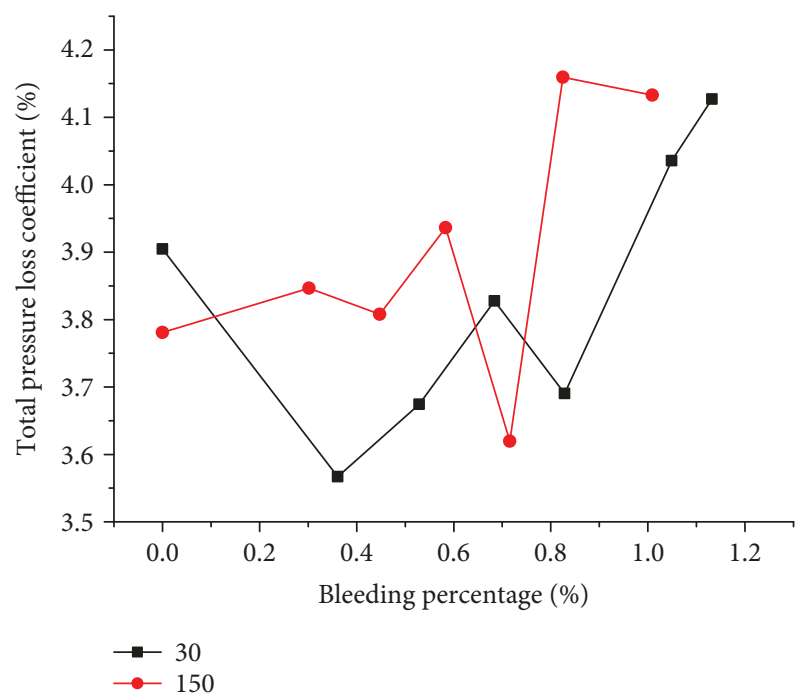

FIGURE 19: Relationship between the total pressure loss coefficient and the bleeding percentage (1.20 expansion ratio).

4.3. Effects of Dump Gap Ratio Variation. The dump gap ratio is defined as the proportion of the distance between the prediffuser outlet and the head of the flame tube in the annulus height of prediffuser outlet. The total pressure loss mainly occurs in the dump gap, more precisely, within the vicinity of the flame tube head. As we can see from Figure 23, the total pressure loss always increases after a short reduction with an increasing dump gap ratio. Thus, there will be a minimum total pressure loss and a maximum static pressure recovery in an optimal dump gap ratio.

When the dump gap is wider than the optimal gap, recirculation area grows asymmetrically which needs more energy to maintain it and the overall length of the diffuse will be

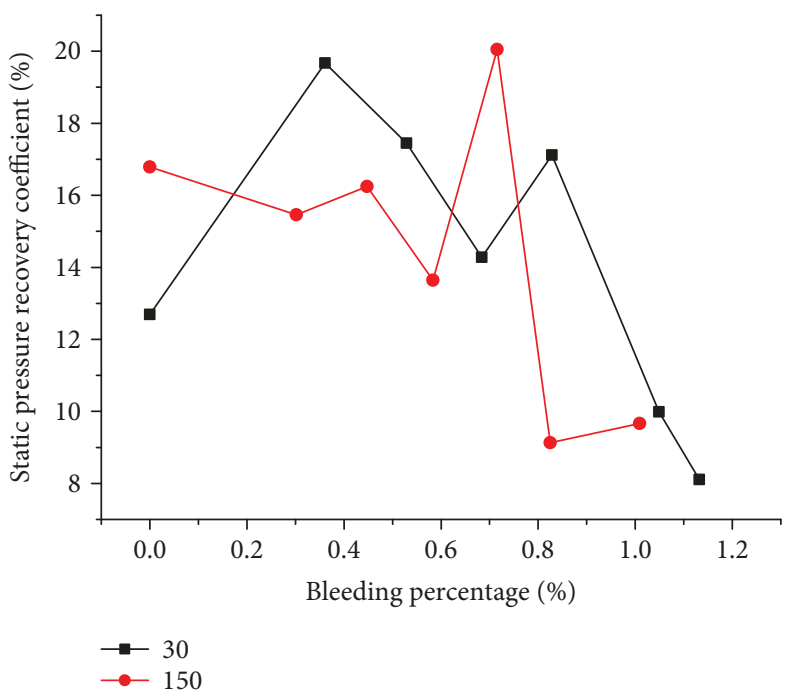

FIgURE 20: Relationship between the static pressure recovery coefficient and the bleeding percentage (1.20 expansion ratio).

longer. The recirculation area might affect the velocity distribution of the prediffuser outlet and flow path in the prediffuser or even block the flow path. The blocked flow path will guide the flow only to pass through one of the two flow paths, inner or outer annulus, depending on the physical model.

In contrast, when a dump gap is narrower than the optimal gap, the streamline curves sharply in the dump region, and the mixing of streamlines is severe, which will cause a bigger total pressure loss as well. Moreover, the static pressure recovery of dump region is close to 0 , even negative, if the dump gap is too narrow. When the prediffuser expansion angle is $9^{\circ}$, the optimal dump gap ratio is around 1.1, at which the total pressure loss is minimal. 


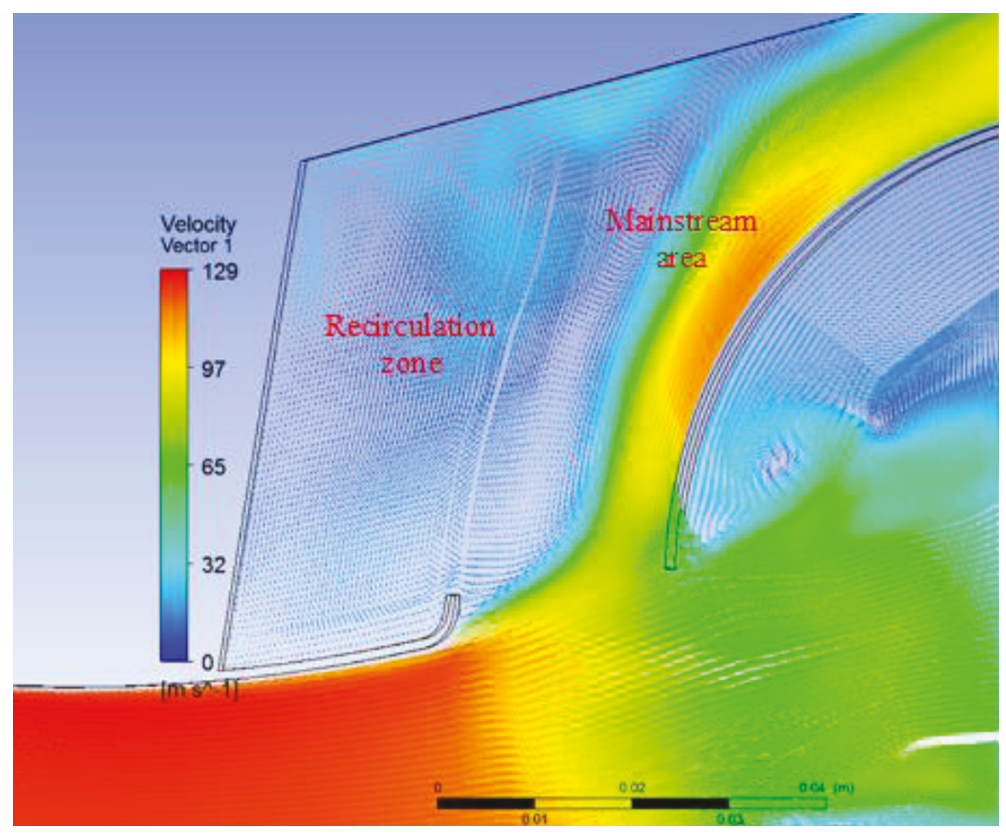

FIGURE 21: Velocity vector.

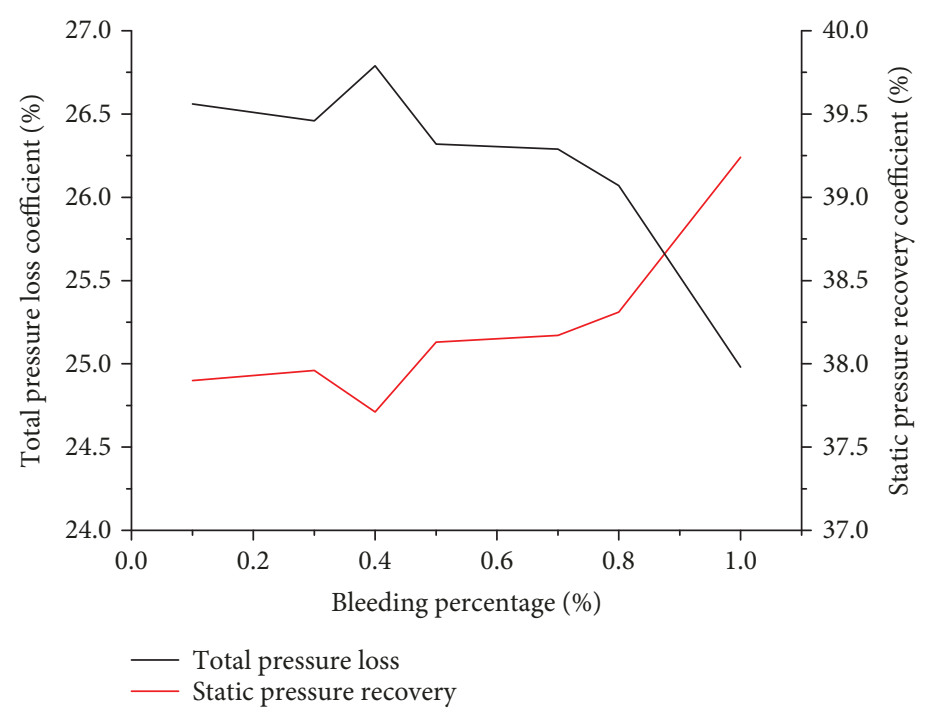

FIgURe 22: Performance with bleeding air from outer dump region.

TABLE 10: Calculation result.

\begin{tabular}{|c|c|c|c|c|c|c|}
\hline Bleeding & Prediffuser $C_{\mathrm{p}}$ & Dump region $C_{\mathrm{p}}$ & Whole $C_{\mathrm{p}}$ & Prediffuser $\Omega$ & Dump region $\Omega$ & Whole $\Omega$ \\
\hline 0 & $30.44 \%$ & $4.65 \%$ & $35.09 \%$ & $5.23 \%$ & $24.22 \%$ & $29.45 \%$ \\
\hline 0.001 & $31.06 \%$ & $8.50 \%$ & $39.56 \%$ & $5.47 \%$ & $19.43 \%$ & $24.90 \%$ \\
\hline 0.003 & $30.85 \%$ & $8.61 \%$ & $39.46 \%$ & $5.46 \%$ & $19.50 \%$ & $24.96 \%$ \\
\hline 0.004 & $31.13 \%$ & $8.66 \%$ & $39.79 \%$ & $5.47 \%$ & $19.24 \%$ & $24.71 \%$ \\
\hline 0.005 & $31.04 \%$ & $8.28 \%$ & $39.32 \%$ & $5.48 \%$ & $19.65 \%$ & $25.13 \%$ \\
\hline 0.007 & $30.84 \%$ & $8.45 \%$ & $39.29 \%$ & $5.46 \%$ & $19.71 \%$ & $25.17 \%$ \\
\hline 0.008 & $30.94 \%$ & $8.13 \%$ & $39.07 \%$ & $5.48 \%$ & $19.83 \%$ & $25.31 \%$ \\
\hline 0.01 & $30.18 \%$ & $7.80 \%$ & $37.98 \%$ & $5.55 \%$ & $20.69 \%$ & $26.24 \%$ \\
\hline
\end{tabular}




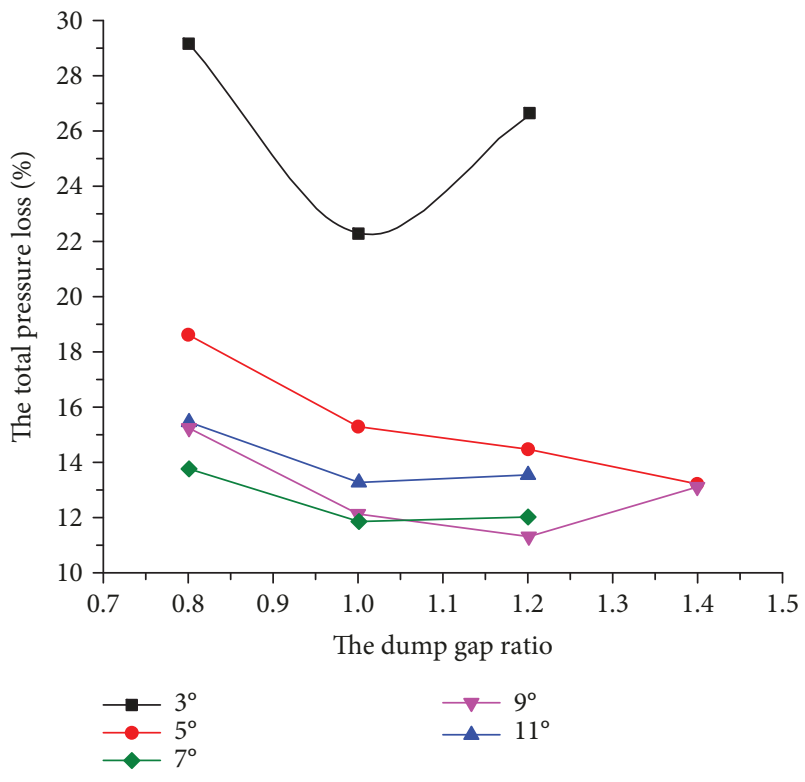

Figure 23: Relationship between the total pressure loss and the dump gap ratio with varieties of prediffuser expansion angle.

\section{Conclusions}

In this paper, experimental and numerical study of the flow in the dump diffuser is presented. A simplified test model is used to investigate the effect of air bleeding on the total pressure loss and the static pressure recovery in dump diffusers. The experimental results show that with the increase of the prediffuser expansion angle, the static pressure recovery coefficient increases and the total pressure loss decreases. The bleeding air from the dump region is beneficial to improve the performance of the dump diffuser, especially when the velocity distribution at the prediffuser outlet is uneven. With the increase of bleeding percentage, the total pressure loss coefficient firstly decreases and then increases, while the static pressure recovery coefficient firstly increases and then decreases. The optimum bleeding percentage is about $0.4 \% \sim 0.7 \%$. The numerical result is in good agreement with the experiment data. When the bleeding percentage is $0.4 \%$, the total pressure loss coefficient is minimum and the static pressure recovery coefficient is maximum.

\section{Nomenclature}

$H$ : $\quad$ Annulus height

$L$ : $\quad$ Length of prediffuser

$\mathrm{H}_{2} / \mathrm{H}_{1}$ : Prediffuser expansion ratio

$m$ : $\quad$ Mass flow rate

$D: \quad$ Dump gap (distance between head of flame tube and outlet of prediffuser)

S: $\quad$ Flow split

$P: \quad$ Static pressure

$P_{\mathrm{t}}: \quad$ Total pressure

$W: \quad$ Width of flame tube

$U_{0}$ : Inlet velocity of the prediffuser

$U$ : $\quad$ Local axial velocity $x$ : Horizontal distance to prediffuser inlet

$y: \quad$ Vertical distance to inner annulus wall

$\rho: \quad$ Fluid density

$\Omega: \quad$ Total pressure loss coefficient

$C_{\mathrm{p}}: \quad$ Pressure recovery coefficient.

\section{Subscripts}

1: Prediffuser inlet

2: $\quad$ Prediffuser outlet

3/4: Outer/inner annulus flow field.

Superscripts

-: Mass-weighted mean value.

\section{Conflicts of Interest}

The authors declare that they have no conflicts of interest.

\section{Acknowledgments}

This paper is written with the experimental help from the Engine Combustion Laboratory of Nanjing University of Aeronautics and Astronautics and Fluid Mechanics and PIV Laboratory of Xiamen University. The authors would like to thank Professor Bao Feng and his research team for their inspirational discussions on the research. The authors are also grateful to the Fundamental Research Funds from the Shenyang Engine Research Institute for providing the funding support for the research.

\section{References}

[1] C. R. Fishenden and S. J. Stevens, "Performance of annular combustor-dump diffusers," Journal of Aircraft, vol. 14, no. 1, pp. 60-67, 1977.

[2] S. J. Stevens, U. S. L. Nayak, J. F. Preston, P. J. Robinsons, and C. T. J. Scrivener, "Influence of compressor exit conditions on diffuser performance," Journal of Aircraft, vol. 15, no. 8, pp. 482-488, 1978.

[3] S. Stevens, A. Wray, and P. Price, "The aerodynamic performance of a modern vaporizing combustor dump diffuser," in 24th Joint Propulsion Conference, Boston, MA, USA, July 1988.

[4] A. Klein, "The relation between losses and entry-flow conditions in short dump diffusers for combustors," The Aeronautical Journal, vol. 92, no. 920, pp. 390-396, 1988.

[5] A. Klein, "Characteristics of combustor diffusers," Progress in Aerospace Sciences, vol. 31, no. 3, pp. 171-271, 1995.

[6] T. Zierer, "Experimental investigation of the flow in diffusers behind an axial flow compressor," Journal of Turbomachinery, vol. 117, no. 2, p. 231, 1995.

[7] A. G. Barker and J. F. Carrotte, "Influence of compressor exit conditions on combustor annular diffusers, part 1: diffuser performance," Journal of Propulsion and Power, vol. 17, no. 3, pp. 678-686, 2001.

[8] A. G. Barker and J. F. Carrotte, "Influence of compressor exit conditions on combustor annular diffusers part II: flow redistribution," Journal of Propulsion and Power, vol. 17, no. 3, pp. 687-694, 2001. 
[9] J. F. Carrotte, D. W. Bailey, and C. W. Frodsham, "Detailed measurements on a modern combustor dump diffuser system," Journal of Engineering for Gas Turbines and Power, vol. 117, no. 4, pp. 678-685, 1995.

[10] R. Hestermann, S. Kim, A. Ben Khaled, and S. Wittig, "Flow field and performance characteristics of combustor diffusers: a basic study," Journal of Engineering for Gas Turbines and Power, vol. 117, no. 4, pp. 686-694, 1995.

[11] S. Honami, T. Shizawa, A. Sato, and H. Ogata, "Flow behavior with an oscillating motion of the impinging jet in a dump diffuser combustor," Journal of Engineering for Gas Turbines and Power, vol. 118, no. 1, pp. 65-71, 1996.

[12] A. D. Walker, J. F. Carrotte, and J. J. McGuirk, "The influence of dump gap on external combustor aerodynamics at high fuel injector flow rates," Journal of Engineering for Gas Turbines and Power, vol. 131, no. 3, article 031506, 1733 pages, 2009.

[13] V. R. Sanalkumar, A. Muraleedharan, Y. Khan, A. A. Swamy, and R. Gemson, "Studies on dump diffusers for modern aircraft engines," in 43rd AIAA/ASME/SAE/ASEE Joint Propulsion Conference \& Exhibit, Cincinnati, OH, USA, July 2007.

[14] K. C. Karki, V. L. Oechsle, and H. C. Mongia, "A computational procedure for diffuser-combustor flow interaction analysis," Journal of Engineering for Gas Turbines and Power, vol. 114, no. 1, pp. 1-7, 1992.

[15] A. J. Juhasz, Performance of a Short Annular Dump Diffuser Using Wall Trailing-Edge Suction, NASA Technical Report, NO. NASA-TM-X-3093, 1974.

[16] A. H. Lefebvre, "The role of fuel preparation in low-emission combustion," Journal of Engineering for Gas Turbines and Power, vol. 117, no. 4, pp. 617-654, 1995.

[17] L. L. Xu, C. Ruan, X. Y. Fang, F. Xing, and C. L. Zhao, "Effects of pre-diffuser on performance of dump diffuser," Tuijin Jishul Journal of Propulsion Technology, vol. 36, no. 7, pp. 10541061, 2015.

[18] P. He, J. Suo, K. Xie, S. Chen, S. Shen, and Q. Zeng, "The influence of dump gap on aerodynamic performance of a lowemission combustor dump diffuser," in ASME Turbo Expo 2013: Turbine Technical Conference and Exposition. Volume 1B: Combustion, Fuels and Emissions, San Antonio, Texas, USA, June 2013.

[19] S. Shen, P. He, M. Shang, and R. Mao, "The effects of cowling geometry, area ratio and dump gap on a combustor diffusion system," in ASME Turbo Expo 2014: Turbine Technical Conference and Exposition. Volume 4B: Combustion, Fuels and Emissions, Düsseldorf, Germany, June 2014.

[20] P. Sathyan, S. Srikanth, I. Dheepan, M. Arun, C. Aswin, and V. R. Sanal Kumar, "Studies on aerodynamic characteristics of dump diffusers for modern aircraft engines," Applied Mechanics and Materials, vol. 232, pp. 246-251, 2012.

[21] P. Ghose, A. Datta, and A. Mukhopadhyay, "Effect of prediffuser angle on the static pressure recovery in flow through casing-liner annulus of a gas turbine combustor at various swirl levels," Journal of Thermal Science and Engineering Applications, vol. 8, no. 1, article 011017, 2016.

[22] M. N. Bhat, R. K. Mishra, R. D. Navindgi, and T. K. Sampathkumaran, "Effect of diffuser geometry on the performance of a short annular aero gas turbine combustor," in National Conference on Ic Engine \& Combustion, Bangalore, India, 2001.
[23] L. Xu, Y. Huang, C. Ruan, and P. W. F. Xing, "Study of the dump diffuser optimization for gas turbine combustors," Procedia Engineering, vol. 99, pp. 828-834, 2015.

[24] H. Tennekes and J. Lumley, A First Course in Turbulence, MIT Press, 1972.

[25] A. W. Date, Introduction to Computational Fluid Dynamics, vol. 20, no. 5p. iv, Cambridge University Press, 2005.

[26] P. Bradshaw, The Effects of Streamline Curvature on Turbulent Flow, École Polytechnique Fédérale de Lausanne, 1973.

[27] H. Mongia, G. Hsaio, D. Burrus, P. Sreedhar, A. Rao, and P. Naik, "Combustor diffuser modeling part I: inlet profiles \& 2-D calculations," in 40th AIAA/ASME/SAE/ASEE Joint Propulsion Conference and Exhibit, Fort Lauderdale, Florida, 2013.

[28] A. D. Walker, P. A. Denman, and J. J. McGuirk, "Experimental and computational study of hybrid diffusers for gas turbine combustors," Journal of Engineering for Gas Turbines and Power, vol. 126, no. 4, p. 717, 2004.

[29] A. D. Walker, J. F. Carrotte, and P. A. Denman, "Annular diffusers with large downstream blockage effects for gas turbine combustion applications," Journal of Propulsion and Power, vol. 27, no. 6, pp. 1218-1231, 2011. 


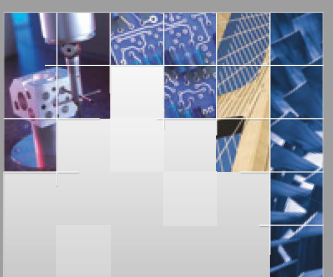

\section{Enfincering}
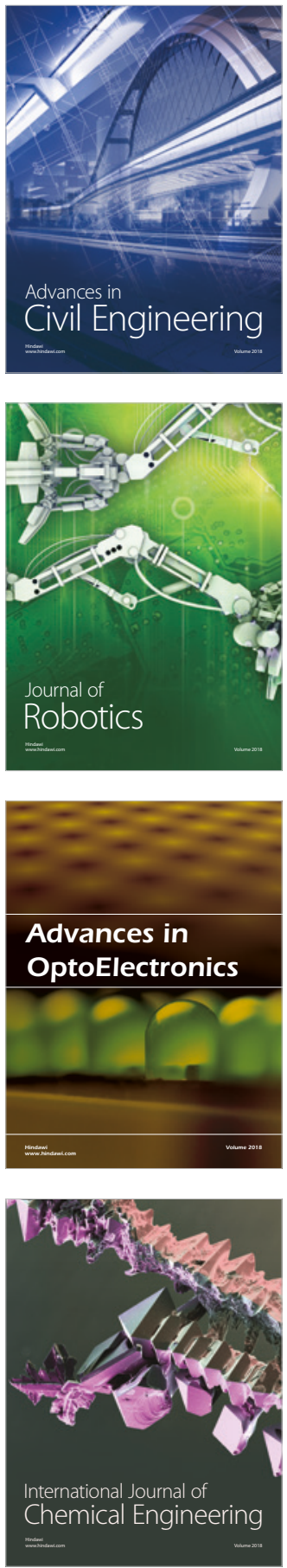

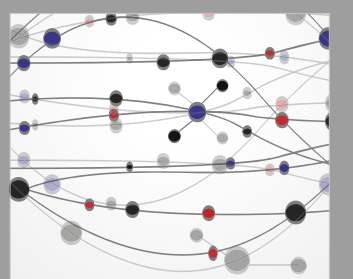

\section{Rotating \\ Machinery}

The Scientific World Journal

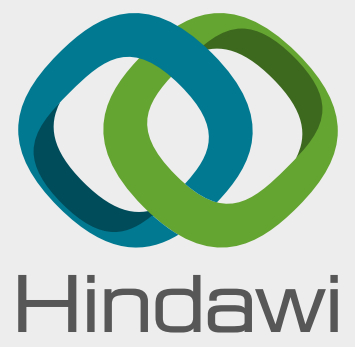

Submit your manuscripts at

www.hindawi.com
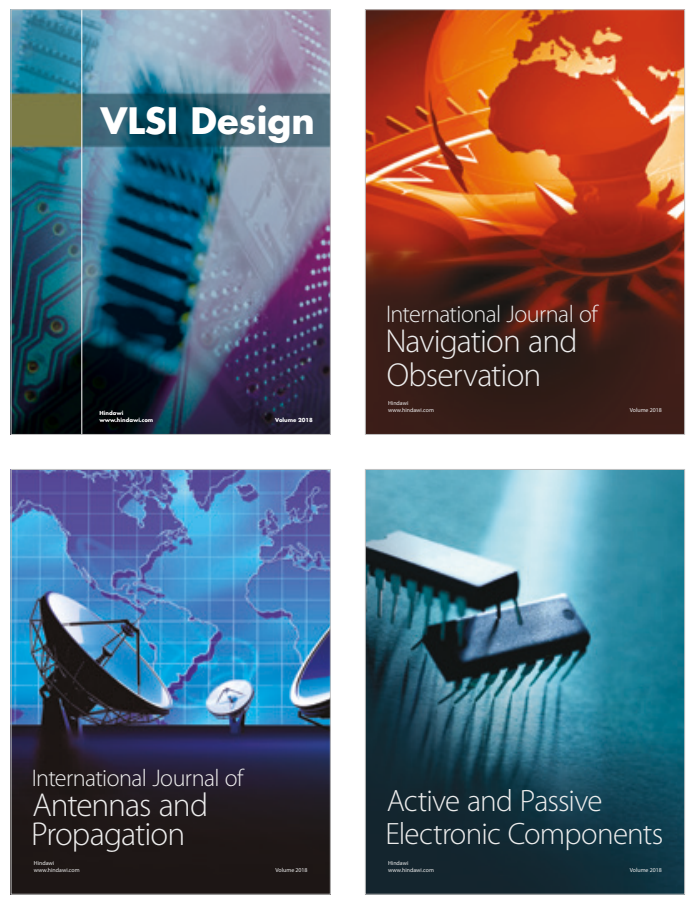
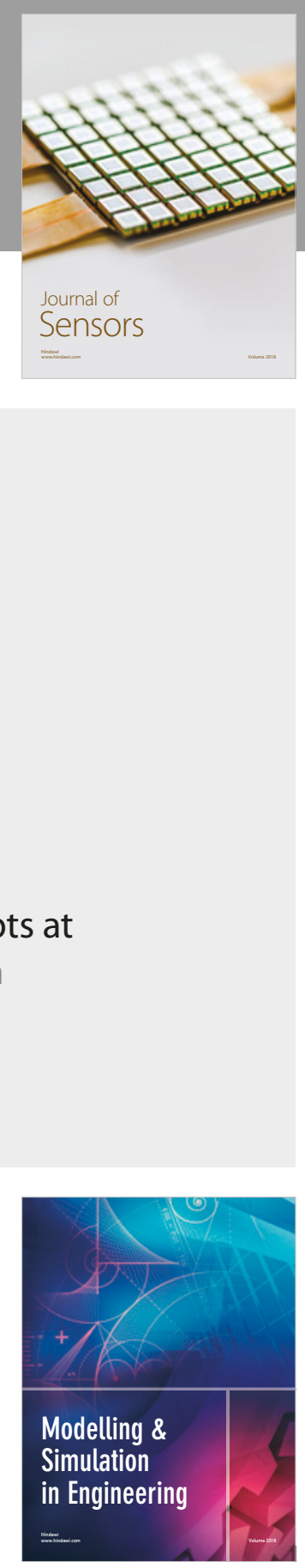

\section{Advances \\ Multimedia}
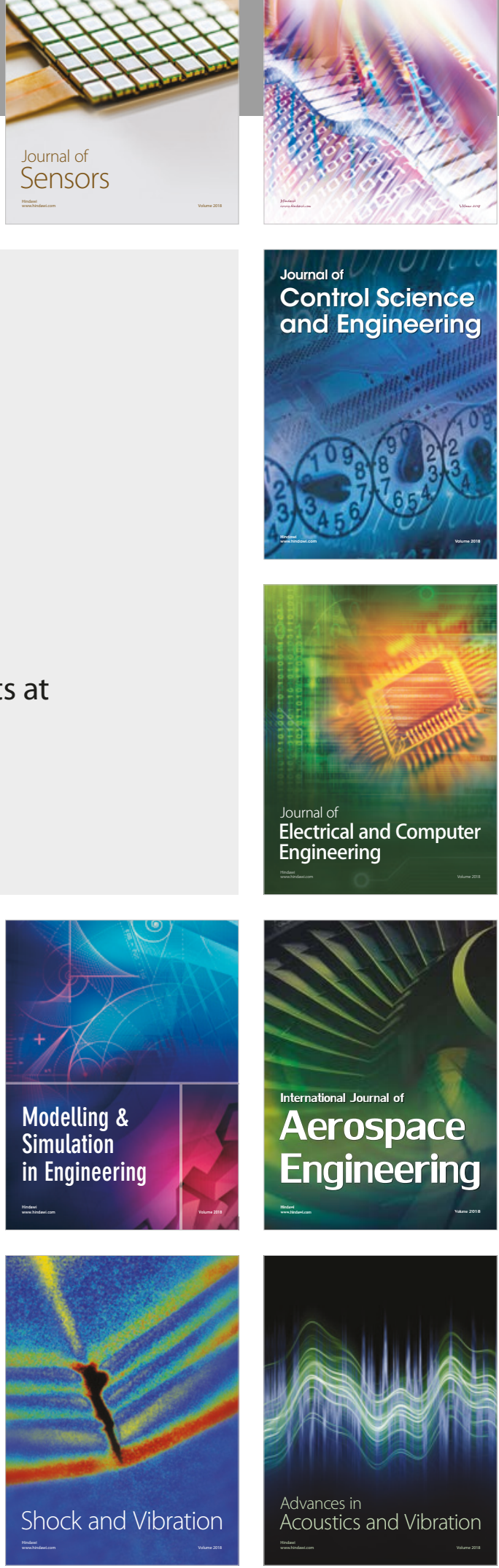